\title{
Reflets
}

Revue ontaroise d'intervention sociale et communautaire

\section{La transformation des institutions et des communautés francophones face à l'immigration et à la mondialisation : une étude de cas}

\section{Amal Madibbo et Normand Labrie}

Volume 11, numéro 1, 2005

Exclusion sociale

URI : https://id.erudit.org/iderudit/013059ar

DOI : https://doi.org/10.7202/013059ar

Aller au sommaire du numéro

Éditeur(s)

Reflets : Revue ontaroise d'intervention sociale et communautaire

ISSN

1203-4576 (imprimé)

1712-8498 (numérique)

Découvrir la revue

Citer cet article

Madibbo, A. \& Labrie, N. (2005). La transformation des institutions et des communautés francophones face à l'immigration et à la mondialisation : une étude de cas. Reflets, 11(1), 49-80. https://doi.org/10.7202/013059ar

Tous droits réservés (C) Reflets : Revue ontaroise d'intervention sociale et communautaire, 2005
Ce document est protégé par la loi sur le droit d'auteur. L'utilisation des services d'Érudit (y compris la reproduction) est assujettie à sa politique d'utilisation que vous pouvez consulter en ligne. 


\section{La transformation des institutions et des communautés francophones face à l'immigration et à la mondialisation : une étude de cas}

Amal Madibbo et Normand Labrie

Centre de recherches en éducation franco-ontarienne, Université de Toronto

\section{Introduction}

Dans le contexte actuel de la mondialisation, plusieurs phénomènes de changement social sont à l'œuvre. D'une part, la mobilité géographique et socio-économique des individus liée aux phénomènes de l'immigration et de l'urbanisation produit d'importants changements démographiques. D'autre part, l'idéologie de l'État providence fait place à une approche néolibérale du rôle de l'État. Ces divers phénomènes de changement ont forcé les organismes sociaux et communautaires servant de liens entre des individus et des institutions, à revoir leurs mandats et leurs pratiques.

Dans le monde francophone au Canada anglais, de telles institutions se sont transformées avec le temps. On est passé d'organismes jouant un rôle de représentation symbolique et de services destinés à une communauté homogène d'origine canadienne-française et européenne vers des agences qui offrent 
"La francophonie torontoise, par exemple, compte aussi bien des gens originaires du MoyenOrient, d'Afrique, d'Asie, des Caraïbes, que d'Europe de l'Est. » des services à une clientèle de diverses origines géographiques et sociales et ayant diverses pratiques langagières et identitaires (Berger 1997; Madibbo et Maury 2002).

Un organisme communautaire peut cumuler une fonction symbolique de ralliement et d'identification, une fonction communautaire de regroupement de par ses activités, et une fonction de pourvoyeur de services répondant aux besoins des usagers, membres des communautés. L'organisme communautaire idéal en milieu francophone minoritaire cumulerait les trois fonctions. Comme nous le verrons dans cet article, des tensions émergent entre ces différentes fonctions et des contradictions apparaissent dans leur mise en œuvre. Sur le plan démographique, ces tensions relèvent de la transformation des populations francophones liée à l'immigration et à la mobilité socio-économique propre à l'urbanisation. C'est-à-dire que la population francophone s'est transformée d'une collectivité surtout d'origine canadiennefrançaise et européenne de classe moyenne en une population très diversifiée sur le plan des origines géographiques et sociales. La francophonie torontoise, par exemple, compte aussi bien des gens originaires du Moyen-Orient, d'Afrique, d'Asie, des Caraïbes, que d'Europe de l'Est. Il y a des gens appartenant aux diverses classes sociales et engagés dans des processus de changement de statut socio-économique, soit ascendant, soit descendant. Sur le plan du soutien des organismes par l'État, ces tensions relèvent également d'une politique de financement dispersée, aux objectifs ciblés et limités dans le temps. Ainsi, conformément à une approche néolibérale, l'État procède à une dévolution de ses responsabilités vers des organismes communautaires, en imposant à ces derniers un cadre de contrôle d'imputabilité et de redevabilité.

L'objectif de cet article est d'expliquer comment l'un de ces organismes francophones en milieu minoritaire s'est adapté au changement social et à la mondialisation en se transformant d'un centre culturel avec mission de servir de point de ralliement d'une communauté conçue comme homogène, en un centre d'accueil offrant des services à une clientèle linguistiquement hétérogène et provenant de divers pays du monde francophone. Afin de mieux répondre à ce mandat, l'organisme a dû réviser 
sa raison d'être, axée sur la langue commune d'une population d'origine canadienne-française et européenne, pour tenir compte de pratiques langagières individuelles et institutionnelles plus diversifiées. En outre, la raison d'être de l'organisme a dû être révisée afin que l'organisme demeure un pôle d'identification communautaire.

Ce que nous chercherons à comprendre plus précisément au moyen d'une analyse du discours, c'est comment ces changements se sont concrétisés dans cet organisme d'accueil francophone situé dans un milieu à la fois multiculturel et anglo-dominant comme Toronto, et comment l'organisme s'est adapté à ces changements. Nous nous pencherons sur le cas du Franco-club ${ }^{1}$ en analysant les changements opérés dans la définition de son mandat et dans son fonctionnement, en lien, notamment, avec les services offerts dans les domaines du logement et de la recherche d'emploi. Nous nous attarderons également sur l'adaptation de cet organisme aux pratiques langagières et identitaires de ses usagers eu égard aux langues française, anglaise et autres.

\section{Contexte}

Une multitude d'institutions et d'organismes francophones à Toronto ont été créés au cours des trente dernières années. L'organisme dont il est ici question, le Franco-club, est la résultante des luttes menées dans les années 60/70 par des militants francophones afin d'obtenir leurs propres infrastructures à titre de groupe de langue officielle, parallèles à celles du groupe majoritaire de langue anglaise. Comme d'autres organismes francophones, le Franco-club, a dû réviser sa raison d'être et son fonctionnement au cours des années 90, compte tenu, d'une part, des nouvelles réalités démographiques de Toronto et, d'autre part, de la transformation du rôle de l'État.

La clientèle pour laquelle cet organisme a été fondé, celle d'origine canadienne-française ou européenne, se prévaut très peu de ses services. À la place, on trouve une clientèle d'origines 
ethnoculturelles diversifiées, dont les besoins differrent tout à fait de ceux de la clientèle visée originalement. Conçu comme centre culturel et un regroupement d'organismes francophones, le Franco-club est peu à peu devenu un guichet de services pour les nouveaux arrivants et immigrants, proposant, pour ne donner que quelques exemples, de l'aide au logement et à la recherche d'emploi ou des services légaux liés à l'immigration (Chambon et al. 2001).

Plus récemment, les communautés immigrantes ont commencé à former de nouveaux organismes et regroupements offrant des services semblables à ceux offerts par le Franco-club, mais visant plus précisément les membres de leurs propres communautés. Ces nouveaux organismes se trouvent souvent en compétition avec les organismes établis tel le Franco-club lorsqu'il s'agit d'être reconnus comme porte-parole légitimes de segments de la population francophone. Or cette reconnaissance est nécessaire pour avoir accès aux ressources matérielles sous forme de subventions publiques.

D'un côté, l'État établit des critères et des normes pour avoir accès à ses programmes de financement. De l'autre, les organismes comme le Franco-club tentent de s'adapter tant bien que mal aux exigences administratives. Par contre, les besoins des usagers ne coïncident pas nécessairement avec les services qui en résultent. Comme nous le verrons, les programmes d'accès à l'emploi offerts par le Franco-club visent surtout des personnes à la recherche d'emplois d'insertion du type « col blanc moyen » et, par conséquent, ils ne répondent pas aux besoins de l'ensemble de sa clientèle qui cherche des emplois de divers types. Par ailleurs, les programmes offerts aux nouveaux arrivants doivent répondre à des exigences administratives établies par les organismes gouvernementaux; or, il n'existe pas d'adéquation parfaite entre les besoins des nouveaux arrivants francophones et les normes gouvernementales de redevabilité, d'où un certain mécontentement chez les francophones quant aux services offerts par le Franco-club.

Ce mécontentement se trouve amplifié par un questionnement récurrent sur la raison d'être du Franco-club à l'origine en tant 
que centre communautaire et culturel, et en tant que point central de ralliement de tous les francophones de Toronto. Ainsi, ceux qui ont d'autres besoins d'emploi, d'activités culturelles ou politiques peuvent à la limite les obtenir en milieu anglophone ou par le biais des associations ethnoculturelles. De ce fait, le Franco-club devrait également fidéliser une population hétérogène et offrir des services à une clientèle ayant divers besoins et des pratiques langagières plus diversifiées que celles envisagées au départ.

Dans un tel contexte, l'étude de cas du Franco-club devrait nous permettre d'expliquer comment le changement social et démographique affecte le fonctionnement des institutions et inversement, comment ces institutions se transforment pour assurer leur existence et leur légitimité. Pour ce faire, nous procéderons à une analyse du discours, dont nous préciserons les fondements théoriques et les approches méthodologiques dans les deux prochaines sections.

\section{Fondements théoriques}

Dans des travaux antérieurs, Heller et Labrie (2003) ont cherché à identifier les espaces dans lesquels des francophones du Canada se constituaient comme communautés de langue minoritaire, comment ces espaces étaient formés, maintenus, reproduits et transformés, quels rapports les individus entretenaient aux institutions, et vice versa. Pour ce faire, ils ont eu recours à une analyse des discours en circulation dans ces espaces.

Heller et Labrie (2003) considèrent que les réalités sociales sont construites au moyen du discours. Ainsi, les acteurs sociaux élaborent des thématisations, ou formations discursives, pour employer le terme proposé par Foucault (1969), concernant les réalités sociales qui les entourent. C'est aussi par le discours que les acteurs sociaux formulent leurs propres positionnements par rapport à ces réalités sociales (Mondada 1998). Ces prises de position d'un acteur social au sujet d'une réalité en particulier tiennent compte de la place qu'il occupe dans la société, soit comme individu, comme membre 
d'une communauté, agent institutionnel, ou représentant de l'État, et de sa trajectoire individuelle.

Afin de comprendre comment des communautés francophones en milieu minoritaire s'adaptent au changement social en général, et en particulier aux transformations découlant de la mondialisation, de l'immigration et de l'urbanisation, Heller et Labrie (2003) ont développé une approche théorique consistant à traiter des discours en circulation, compte tenu de l'évolution des politiques sociales, des rapports de pouvoir, des stratégies de reproduction sociale et linguistique des communautés francophones et de la transformation du rôle de l'État. Trois types de discours ont été identifiés, à savoir le discours traditionaliste, misant sur une conception de la communauté homogène, repliée sur l'église et la paroisse; le discours modernisant, comptant sur l'État providence pour l'établissement d'organismes francophones autonomes, où l'affiliation à la communauté ethnolinguistique demeure le point de repère fondamental; finalement, le discours mondialisant, répondant aux idéologies néo-libérales selon lesquelles l'économie a prédominance sur le politique et l'allégeance à la communauté linguistique n'est plus aussi centrale, vu les identités multiples. Heller et Labrie soutiennent que ces trois types de discours coexistent présentement dans les divers espaces au sein desquels la francophonie est définie.

En nous inspirant de cette approche théorique, nous mettrons l'accent, dans l'analyse qui suit, sur les discours modernisant et mondialisant et nous tenterons d'expliquer les discours individuels et institutionnels circulant dans des institutions et communautés francophones produits par les acteurs sociaux afin de composer avec des phénomènes de changement social. Notre analyse du discours consistera alors à comprendre et à expliquer ces pratiques quant à la spécificité de l'accès aux ressources symboliques et matérielles, l'impact de l'immigration sur le fonctionnement des institutions francophones, et à dégager les tensions et les contradictions émanant des divers interlocuteurs en fonction de leur positionnement.

En ce qui concerne les pratiques des communautés et institutions francophones à Toronto quant à la minoration et aux services sociaux, la coexistence de ces deux derniers types 
de discours met en lumière des contradictions dans la définition même des communautés francophones, qui se répercutent sur la conception de la raison d'être et du fonctionnement des institutions francophones (Labrie et al.2002). Ainsi, le cas des services sociaux nous semble important, car il permet de saisir ces dynamiques au sein des communautés francophones, et le lien entre les idéologies nationalistes, leurs manifestations institutionnelles et leurs capacités de composer avec la diversité et les pratiques bilingues et multilingues des usagers définis comme clients. Nous sommes ici à la jonction des discours modernisant et mondialisant. Dans la prochaine section, nous verrons de quelle façon nous avons appliqué cette approche axée sur l'analyse du discours pour notre étude sur les transformations des communautés et des institutions francophones en milieu minoritaire.

\section{Approche méthodologique}

Les données sur lesquelles nous nous basons ont été recueillies dans le cadre du projet l'immigration et la communauté franco-torontoise ${ }^{2}$ dont l'objectif était de comprendre l'expérience des immigrants francophones en milieu minoritaire à Toronto et d'examiner l'impact de leur arrivée sur l'évolution des structures d'accueil franco-torontoises. Pour ce faire, nous avons mené une recherche de type ethnographique basée sur des entrevues, des séances d'observation et une analyse documentaire. Cette recherche a été menée auprès de deux organismes, ou agences, et de deux groupes de nouveaux arrivants.

Les deux organismes étudiés comprennent le Franco-club, qui fait l'objet de cet article, une agence spécialisée dans les conseils à l'emploi et autres services aux nouveaux arrivants, et la Francoclinique spécialisée dans les services de santé. Nous avons réalisé des entrevues avec le personnel clé de ces deux agences; dans le cas du Franco-club, il s'agit de quatorze personnes, comprenant en fait la quasi-totalité du personnel, et dans le deuxième cas, il s'agit de onze personnes, ou la moitié du personnel. Les participants ont été 
sélectionnés en accord entre l'équipe de recherche et la direction des agences dans le but de parvenir à un échantillon représentatif des divers services offerts, des diverses catégories de personnel et des différents groupes d'origine canadienne-française, européenne, haïtienne et africaine. Ces entrevues ont porté sur les services auprès de nouveaux arrivants et immigrants francophones, sur les rapports entre cette clientèle et celle établie depuis plus longtemps à Toronto, sur l'évolution de la clientèle et de ses besoins, sur l'évolution des liens entre l'agence et les bailleurs de fonds surtout gouvernementaux, et sur le recrutement du personnel. Nous avons également posé des questions sur les trajectoires professionnelles des personnes interviewées. Finalement, nous avons pu réaliser trois séances d'observations sur place, incluant une activité et des réunions du personnel de la seconde agence.

Pour ce qui est des deux groupes de nouveaux arrivants, nous avons réalisé des entrevues auprès de quatorze personnes d'origine haïtienne et quatorze d'origine mauricienne, dont plusieurs entrevues collectives auprès de membres de regroupements communautaires. Nous nous sommes limités à deux groupes pour des raisons de faisabilité, puisqu'il nous fallait avoir une compréhension approfondie des caractéristiques sociales des groupes et de leurs trajectoires de migration. Nous avons donc choisi deux groupes qui se distinguaient en fonction de la trajectoire migratoire et des pratiques langagières, culturelles et religieuses. Le groupe haïtien est implanté depuis plusieurs décennies à Toronto, et la plupart de ses membres sont d'abord passés par le Québec. En général, ces derniers parlent le français et le créole et, une fois installés à Toronto, ils doivent faire l'acquisition de l'anglais. Une première vague migratoire de l'élite formée de la classe moyenne a été suivie d'autres vagues de gens de condition plus modeste, pratiquant le catholicisme et le vaudouisme. Le groupe mauricien est arrivé plus récemment et est venu directement de l'île Maurice. Il est composé en majorité de gens d'origine sino- et hindo-mauricienne parlant généralement le français, l'anglais, le créole mauricien, et le hakka dans le cas des Mauriciens d'origine chinoise. Il s'agit, dans bien des cas, de gens issus de la classe moyenne, souvent des commerçants, ayant quitté 
"Cette analyse devrait nous permettre de saisir comment les institutions des communautés francophones composent avec les nouvelles réalités liées à la mondialisation et en quoi le cas des institutions francophones, axées sur la base de l'identité linguistique, se démarquent des autres institutions. " leur pays à la suite de l'indépendance. Plusieurs sont catholiques, ce qui renforce leurs liens avec des institutions francophones.

Notre échantillon se constitue d'individus, dont un petit nombre étaient aussi des clients des deux agences participant à l'étude, et de représentants de regroupements communautaires qui jouent un rôle public important dans leur communauté ainsi que dans les autres organismes torontois. Les entrevues ont porté sur les trajectoires d'immigration, l'utilisation des services offerts par les organismes francophones, les rapports avec les institutions de la francophonie torontoise, ainsi qu'avec les organismes de langue anglaise et ceux de la communauté d'origine, les modes d'organisation interne de la communauté, et ses liens avec diverses communautés à Toronto. Nous nous sommes également concentrés sur la formation et l'éducation, la trajectoire linguistique, et la perception de la francophonie torontoise. Les participants ont été sélectionnés selon la date d'arrivée à Toronto, ce qui nous a permis de saisir les divers positionnements vis-à-vis les deux agences.

De plus, nous avons pu effectuer deux séances d'observation dans des regroupements mauriciens. Finalement, les deux organismes et deux communautés nous ont généreusement fourni l'accès à un certain nombre de documents internes et publics pertinents, comme des études, des rapports internes, des plans stratégiques de développement, des procès-verbaux ou des bulletins d'information.

En nous basant sur le discours produit au cours d'entrevues auprès des responsables et clients du Franco-club, ainsi que des membres des deux communautés, nous examinons brièvement les éléments suivants : le changement du rôle et du fonctionnement du Franco-club, l'émergence des discours modernisants et mondialisant tels qu'identifiés par Heller et Labrie (2003), le paradoxe entre la réorganisation des services telle que prévue par l'État, d'une part, et les besoins des clients, d'autre part, ainsi que l'adaptation de cet organisme aux pratiques langagières et identitaires de ses usagers liées à l'usage des langues française, anglaise et autres. Cette analyse devrait nous permettre de saisir comment les institutions des communautés francophones 
composent avec les nouvelles réalités liées à la mondialisation et en quoi le cas des institutions francophones, axées sur la base de l'identité linguistique, se démarquent des autres institutions. Ainsi, nous comprendrons mieux pourquoi et comment des institutions sociales se transforment, et quelles en sont les conséquences et pour qui. Voyons d'abord comment le mandat du Franco-club s'est transformé au cours des dernières années.

\section{Le Franco-club face au changement social et à la transformation du rôle de l'État}

Né dans les années soixante-dix du regroupement de divers organismes francophones à vocation communautaire et culturelle, le Franco-club se voulait à l'origine un centre communautaire et culturel au service des francophones de Toronto composés essentiellement d'Ontariens, de Québécois et d'Européens. Cette vocation a pu se développer dans un premier temps grâce à l'appui du gouvernement fédéral canadien intéressé à promouvoir sa vision pancanadienne du bilinguisme, et grâce au gouvernement provincial aussi longtemps que ce dernier a participé activement au financement d'activités culturelles. Cet organisme a dû réorienter son fonctionnement au cours des années quatre-vingt-dix, compte tenu, d'une part, des nouvelles réalités démographiques et, d'autre part, des nouvelles règles de financement. Dans les années quatrevingt-dix, les appuis gouvernementaux se sont estompés. La clientèle s'est transformée pour inclure davantage de nouveaux arrivants et immigrants des communautés ethnoculturelles, ayant des besoins différents. Dans l'une des entrevues, M. Morin, un responsable de l'organisme soulève cette question :

Monica : Les francophones de souche vont demander quoi?

M. Morin : Des activités sociales et communautaires [...]. Ils vont demander du support dans certaines 
activités, mais ils ont déjà des institutions, les conseils scolaires [et] ils ont déjà leurs propres institutions [...], ils ont déjà leurs paroisses.

Monica. : Et pour les nouveaux arrivants, ce serait plutôt emploi?

M. Morin: Les nouveaux arrivants, c'est les services légaux, services de réfugiés, services d'immigration, les services d'accompagnement dans la recherche de logement [...], le service social [...], et puis l'emploi, bien sûr.

Cet extrait identifie les deux groupes majeurs qui constituent la clientèle; les francophones de souche et les nouveaux arrivants. Ce discours indique que ces deux groupes se distinguent en fonction de leurs besoins et des institutions qui peuvent y répondre. Les francophones de souche ont déjà accès à des institutions au sein desquelles ils exercent un certain pouvoir, et dont certaines créent de l'emploi. Leurs besoins auprès du Franco-club se limitent à l'appui à leurs clubs et réseaux sociaux pour des activités de nature récréative. Par contre, comme l'indique ce discours, les nouveaux arrivants, qui représentent actuellement la majorité de la clientèle du Franco-club, ont besoin des services comme l'orientation en matière d'immigration et l'aide à l'emploi, des besoins donc de nature socio-économique, c'est-à-dire liés à l'établissement et à l'intégration. Leurs activités culturelles sont plutôt organisées à travers leurs regroupements communautaires.

Le changement de clientèle, en conjonction avec le changement du rôle de l'État, comme nous le verrons plus loin, a amené le Franco-club à repenser son mandat. Ainsi, le Franco-club est devenu avec le temps principalement un guichet de divers services aux nouveaux arrivants. Comme nous le verrons, ce changement est manifeste dans le passage du discours modernisant au discours mondialisant. M. Morin, le même responsable du Franco-club, explique :

"Bien, le [Franco-club], son mandat, c'était plutôt un organisme $[. .$.$] qui chaperonne l'ensemble des$ 
organismes puis qui les appuie puis qui les aide [...] Mais, avec le temps, il a perdu ce rôle-là, puis [...] Là où il $y$ avait de l'argent, c'était dans les services aux nouveaux arrivants, les services d'emploi. Alors, le [Franco-club] s'est orienté vers ça et c'est rapidement devenu la partie la plus importante, le service aux nouveaux arrivants [...] Ça représente $90 \%$ des activités du [Franco-club].»

La première partie de cet extrait ( Ben... aide ») représente bien le discours modernisant qui s'est développé à partir des années soixante. Ce type du discours consiste à accompagner le mouvement d'expansion de l'État moderne, et ainsi à revendiquer des droits comme communauté linguistique et à se créer des espaces institutionnels autonomes soutenus par l'État (Heller et Labrie 2003). Dans le domaine des services sociaux, on voit les gouvernements provinciaux devenir des maîtres d'œuvre qui créent des institutions et des centres culturels. L'État, et plus particulièrement le gouvernement provincial, apparaît alors comme l'interlocuteur cible de l'élite francophone qui développe une argumentation autour du besoin des populations francophones d'obtenir des services sociaux de qualité dans leur langue. Comme le démontre cet extrait, ce discours modernisant donnera des résultats à Toronto avec la mise sur pied de services sociaux et culturels offerts aux communautés francophones (Labrie et al. 2002). Cependant, ces services sont conçus pour une population francophone homogène sur les plans langagier et ethnique.

On voit ici un déplacement des ressources consenties par l'État en raison des changements démographiques, et un ajustement dans les activités de l'organisme, lié aussi bien à ces mêmes changements démographiques qu'aux politiques gouvernementales de financement. Cet acteur social parle du contexte historique et actuel du Franco-club. L'utilisation du terme "mais» démontre l'opposition entre les deux types de mandats de l'organisme. La première partie de cet extrait est bien représentative de l'idéologie modernisante qui fait de l'organisme un rassemblement regroupant l'ensemble des organismes et 
"...la transformation du rôle de l'État, les changements démographiques et les nouvelles approches identitaires linguistiques se reflètent dans les nouvelles stratégies de reproduction sociale et linguistique, incluant dans la conception des services. Les services offerts au Francoclub sont segmentés, chacun dirigé par un département et désigné à une catégorie spécifique de clientèle. » associations francophones. Il remplit une fonction symbolique de ralliement et d'identification et est reconnu comme porte-parole légitime de la population francophone dans son ensemble. Les services de cet organisme sont offerts en français. En effet, c'est ce rôle-là que le Franco-club jouait antérieurement. Il représentait, regroupait et appuyait les organismes et associations francophones. Mais ce mandat de nature modernisante sera graduellement transformé en un mandat de nature mondialisante. De ce fait, la deuxième partie de cet extrait (mais... du [Franco-club]) est de nature mondialisante car il démontre que le nouveau mandant du Franco-Club se manifeste par l'offre des services en fonction du changement démographique qui produit une nouvelle clientèle, celle des nouveaux arrivants. Ce nouveau mandat du Francoclub s'inscrit bien sûr dans le contexte de la mondialisation et de l'idéologie néo-libérale. Conformément à l'idéologie néolibérale, l'État doit réduire ses ressources et leur distribution sous forme d'assistance et d'aménagement des services. De plus, l'État n'est plus l'interlocuteur de l'élite francophone, il octroie des financements pour offrir des services.

Ainsi, l'organisme a pris une nouvelle direction. Le changement s'observe donc du passage d'un organisme de regroupement à un guichet de services. L'immigration a produit un pourcentage élevé de nouveaux arrivants qui représentent la majorité des clients du Franco-club. Ainsi, bon nombre de programmes concernent exclusivement cette clientèle. Il s'agit de services d'accueil, de conseil en immigration, de recherche de logement, de préparation à l'emploi et des cours d'anglais. En plus de la transformation du mandat de l'organisme dans le domaine des services, on observe que l'accent n'est plus mis sur une seule langue, à savoir le français. Comme nous le verrons, le Franco-club a abandonné l'offre de cours d'anglais pour francophones en guise de stratégie pour la clientélisation de nouveaux arrivants francophones, et il a récemment introduit deux langues africaines dans ses services, le lingala et le swahili.

De ce fait, la transformation du rôle de l'État, les changements démographiques et les nouvelles approches identitaires linguistiques se reflètent dans les nouvelles stratégies de reproduction sociale 
et linguistique, incluant dans la conception des services. Les services offerts au Franco-club sont segmentés, chacun dirigé par un département et désigné à une catégorie spécifique de clientèle. Ces services se développent, certains sont d'implantation récente, pendant que d'autres sont élargis ou supprimés. Chaque service fait face à certaines contraintes. En prenant l'exemple de deux services, dont l'un, le logement, est simple et relativement modeste, et l'autre, le service de l'emploi, est complexe, nous verrons comment les services reflètent ces contrastes.

\section{Le service de logement}

Le service de logement a été récemment implanté afin d'aider les nouveaux arrivants, et plus spécifiquement les réfugiés, à surmonter divers obstacles rencontrés pour trouver du logement, dont la difficulté de communiquer en anglais avec les propriétaires. Lois, l'intervenant responsable de ce service, est d'origine africaine. Il explique la raison d'être du service :

"C'est un service qui a été monté exclusivement pour les nouveaux arrivants francophones [...] C'est des personnes d'abord qui viennent d'arriver et de deux qui ne parlent pas anglais [...] Pourquoi? [...] Parce que c'est des personnes qui ne savent pas encore comment se déplacer dans la ville, comment utiliser le transport en commun [...] et deuxièmement les personnes qui ne parlent pas anglais parce que c'est des personnes qui même si ils [elles] savent se déplacer, elles ne sauront pas communiquer ou lire un contrat bail ou comprendre et puis le signer [...] Il faut convaincre le propriétaire parce qu'en fait on se butte à un dilemme extraordinaire parce que les personnes qui viennent d'arriver [...] ne connaissent personne, mais le propriétaire exige d'eux, d'elles plutôt, et c'est les personnes sur l'aide sociale, les propriétaires exigent qu'ils emmènent un cosignataire ou deux mais vous ne connaissez personne ici, vous 
venez d'arriver [...] et puis certains propriétaires [...] ne veulent pas des gens sur l'aide sociale."

Cet extrait représente également le discours mondialisant, car il souligne l'émergence d'une nouvelle catégorie de francophones, celle de nouveaux arrivants, et le nouveau service qu'offre le Franco-club afin de s'adapter à cette nouvelle clientèle et à ses besoins. Dans le discours de cet acteur social, le terme "nouveaux arrivants " désigne une catégorie spécifique de clients qui ne maîtrisent pas l'anglais et qui ne peuvent pas s'orienter dans la ville. Cette catégorie se compose principalement de réfugiés nouvellement arrivés au Canada, seule clientèle éligible à ce service financé par l'État. Le problème de la langue se présente du fait que les propriétaires sont en majorité anglophones. Plusieurs nouveaux arrivants, ne maitrisant pas forcément l'anglais, ont de la difficulté à communiquer en anglais. Le but du service est donc de les aider à entrer en contact avec des propriétaires et de les accompagner dans les rencontres éventuelles. Le service fonctionne suivant un plan d'intervention en faisant un suivi auprès des clients et des propriétaires. De plus, l'organisme fait une campagne de publicité dans les médias sur l'existence du service. Bien que les annonces soient mises en français, les propriétaires qui contactent l'organisme pour signaler qu'ils ont des logements à louer, sont en majorité issus des communautés non francophones. Le rôle de l'intervenant consiste aussi en des négociations avec les propriétaires qui posent parfois des exigences difficiles à remplir pour des nouveaux arrivants.

Ce discours soulève d'autres barrières auxquelles fait face cette clientèle, dont la demande d'amener un cosignataire. Cela représente une difficulté majeure pour un nouvel arrivant, car ce dernier ne connait pas nécessairement quelqu'un qui puisse se porter garant. Ce discours explique aussi que, bien que plusieurs clients reçoivent l'aide sociale, certains propriétaires refusent d'offrir un logement à cette catégorie de gens. D'autres obstacles se présentent. Certains clients sont des personnes seules ou des familles monoparentales dont le revenu est très limité. Or, le coût du loyer est très élevé à Toronto. D'autres, comme des familles 
"...une telle adaptation de l'organisme aux besoins réels de la population est rendue impossible dans l'immédiat du fait que le service dépend d'un contrat de service établi par le gouvernement fédéral pour une durée déterminée de plusieurs mois, et que par ailleurs le renouvellement $d u$ contrat n'est pas assuré. Ainsi, les règles $d u$ financement mettent l'intervenant dans l'incertitude en ce qui concerne la planification $d u$ service. " ayant plusieurs enfants, ont de la difficulté à se faire accepter par certains propriétaires. Ainsi, les règles imposées par ces derniers ne tiennent pas compte de la réalité sociale des nouveaux arrivants. À ce propos, le service vise à aider ces derniers à surmonter ces barrières.

Le service de logement se concentre sur cette catégorie de nouveaux arrivants dont une majorité arrive de l'Afrique centrale ou du Nord. Cependant, il ressort de notre analyse du discours, d'une part, que les nouveaux arrivants haitiens qui répondent aux critères du service ne sont pas nombreux à l'utiliser. Cela peut s'expliquer du fait qu'il s'agit d'une communauté installée à Toronto depuis plusieurs années. Les nouveaux arrivants d'Haïti trouvent des services d'accueil à travers d'autres organismes et réseaux communautaires. D'autre part, les nouveaux arrivants non réfugiés comme les résidents permanents ou les citoyens canadiens de l'extérieur de Toronto qui manifestent un besoin pour un tel service n'y ont pas accès, car les critères de financement étatique ne les incluent pas. Cela a amené l'organisme à prévoir de nouvelles étapes visant l'élargissement du service pour accommoder d'autres catégories de clients. Par contre, une telle adaptation de l'organisme aux besoins réels de la population est rendue impossible dans l'immédiat du fait que le service dépend d'un contrat de service établi par le gouvernement fédéral pour une durée déterminée de plusieurs mois, et que par ailleurs le renouvellement du contrat n'est pas assuré. Ainsi, les règles du financement mettent l'intervenant dans l'incertitude en ce qui concerne la planification du service. On a affaire à un segment de population qui fait face à des problèmes spécifiques, et à un organisme qui tente de trouver des solutions adaptées, en bénéficiant de l'aide de l'État. L'organisme se concentre sur la population cible, mais n'a pas vraiment de contrôle sur les réglementations étatiques ni sur les pratiques des autres acteurs de la société comme les propriétaires. C'est un exemple de l'adaptation au changement entreprise par cet organisme à l'intérieur de contraintes étatiques.

L'examen du service de logement portait en soi sur un secteur d'activité relativement facile à circonscrire, ciblant une clientèle très spécifique. Néanmoins, nous venons de voir que les rapports 
entre la population, le monde communautaire, l'État et la société dans son ensemble sont assez complexes. Dans la prochaine section, nous examinerons des services d'emploi qui visent plusieurs catégories de population, avec des besoins très variés. Nous verrons que le Franco-club trouvera encore des façons de s'adapter, toujours dans un contexte de rapports complexes entre des populations cibles, les organismes et institutions, l'État et la société en général.

\section{Le service d'emploi}

Le service d'aide à l'emploi est ouvert à toutes catégories de clients de divers statuts comme les réfugiés, les résidents permanents et les citoyens canadiens originaires de divers pays, comme l'Égypte, Haït, l'Île Maurice, l'Iran ou la France. Le but du service est de fournir une orientation à l'emploi et de gérer des offres d'emploi émanant des entreprises. Sylvie-Anne, l'une de deux intervenantes en charge du service en explique le fonctionnement :

Mélanie : Alors comment ça se passe? Est-ce que l'employeur appelle ici et fait une demande ou que, et ensuite est-ce que vous aidez les gens ici à préparer leur C.V.ou à faire des entrevues?

Sylvie-Anne :Tout ça madame, il y a beaucoup de travail à faire [...] C'est que, dépendamment du niveau $d u$ besoin, $[\ldots]$ on voit en partant $[\ldots]$ bon, est-ce que vous pouvez me montrer votre C.V?[...] Bien, je suis pas assez fort en anglais pour m'en faire un... Alors là on regarde s'il $y$ a des ressources [...] On évolue le mieux possible. S'il y en a pas de ressource, si la personne elle peut pas, moi je le fais le C.V. avec elle [...] J'explique comment faire un C.V.à tout le monde [...] J'évalue leurs besoins en matière d'emploi.Après ça, je leur donne des informations. S'ils ont besoin de formation sur le plan de la langue ou parfois un peu 
en informatique, c'est souvent ces deux matières qui sont le plus important là [...] en premier la langue, en second l'informatique.

Ce discours est de nature mondialisante parce qu'il souligne la prééminence de l'informatique au marché du travail et parce qu'il nous démontre que le Franco-club commence à offrir des services dans une deuxième langue, l'anglais. Bien que ce service se limite dans ce cas à l'élaboration du curriculum vitae (CV), il attire notre attention au fait que le Franco-club ne se limite plus au français. Cet extrait explique le plan d'intervention qui inclut des démarches spécifiques suivies avec chaque client. La conseillère cerne leurs besoins d'emploi et établit des fiches de renseignements sur les clients. Beaucoup d'importance est accordée au CV que l'intervenante retravaille avec les clients. Le service offre également des conseils sur des aspects comme les techniques d'entrevues et les lettres de couverture et étudie les possibilités d'emploi. Nous pouvons donc remarquer que c'est un processus qui amène les nouveaux arrivants à un stade où ils se familiarisent assez bien avec le système de recherche d'emploi.

Dans cet extrait, la même conseillère mentionne les deux besoins majeurs : la langue anglaise et l'informatique. À cet égard, l'apprentissage de l'anglais est un élément central relié à l'accès à l'emploi, à l'information ainsi qu'à l'adaptation à l'environnement social. Pour identifier les besoins langagiers, le service fait une évaluation linguistique des aptitudes orales et écrites en anglais et en français. L'intervenante explique que, selon les exigences des postes, ceux qui ont besoin d'améliorer leur niveau de langue sont référés à des écoles d'anglais, aux centres d'alphabétisation ou aux collèges. Cela indique que le Franco-club prépare les clients à un marché du travail bilingue. Pour trouver de l'emploi, il faut apprendre l'anglais, mais il faut aussi améliorer son français. Quant à l'informatique, l'organisme dirige les clients vers des écoles appropriées. Comme l'explique Sylvie-Anne, l'intervenante, le défi majeur sur ce plan est que cette formation n'est pas disponible en français : 
" Ça, c'est une demande [...] Si on a des petites plaintes ou des regrets, on va dire, ah, ce n'est pas donné en français nulle part, l'informatique. Mais, c'est souvent les personnes que justement leur niveau est très bas [...] en anglais et puis [...] Ça serait un besoin là [...] Ça fait que je veux dire, on les encourage à avoir leur anglais parce qu'ils ne peuvent pas fonctionner bien ici. "

Le discours de l'intervenante souligne que les clients qui ont besoin d'apprendre l'informatique devraient d'abord acquérir l'anglais. À ce sujet, nous pouvons remarquer que le discours de la personne qui offre le service ne met pas l'accent sur la revendication; le droit des francophones d'avoir accès à un service (en occurrence la formation en informatique) en français. Au contraire, le nouvel arrivant francophone se fait plutôt conseiller d'apprendre l'anglais pour ensuite suivre la formation dans cette langue. Nous pouvons aussi remarquer que pendant que les services sont segmentés, dans le cas présent avec un service spécialisé dans l'emploi, les clients qui sont à la recherche d'emploi ont des besoins multiples et nécessitent l'utilisation spontanée d'autres services ou de formation dans d'autres organismes, comme l'apprentissage de l'informatique ou de l'anglais.

Les clients à la recherche d'emplois sont différents sur d'autres plans, tels que les niveaux de compétence, de formation et de qualification, comme l'indiquent ces propos de Sylvie-Anne :

"Ça peut partir de la personne qui était réceptionniste ou même coiffeuse [...] jusqu'au médecin, facilement [...] On en voit dans tous les domaines, tous les niveaux d'études, très peu à très avancé, des doctorats [...] doyens, même universitaires. "

Ces paroles nous démontrent que, bien que les qualifications et les niveaux des clients soient divers, une proportion importante de personnes sont hautement qualifiées, comme des médecins ou des professeurs. À cet égard, la question de l'emploi soulève la distinction entre ce que les nouveaux arrivants cherchent, et 
ce qu'ils obtiennent comme travail par le biais de l'organisme. Les clients cherchent divers types d'emplois. De plus, il y en a qui veulent immédiatement travailler pour répondre aux besoins urgents de la vie. Comme le souligne l'intervenante, il s'agit des clients qui disent : " Je suis prêt à faire n'importe quoi [...] Je veux travailler [...] J'ai une famille à faire vivre ». Certains visent un emploi mieux rémunéré. Plusieurs, dont ceux qui sont hautement qualifiés, veulent continuer à exercer leur profession. En somme, les gens ont des qualifications diversifiées, et des stratégies de carrière diverses. L'intervenante identifie ce qui est disponible comme travail : "La plupart, en général, ça dépend toujours de la profession, il y a tellement de professions, la plupart, ce qu'on a en gros $[. .$.$] c'est beaucoup le service à la clientèle. Alors, offrir$ des services dans les deux langues".

Tandis que les clients de l'organisme qui sont définis comme francophones cherchent diverses catégories du travail, les emplois disponibles varient mais se concentrent néanmoins dans certains domaines spécifiques, comme celui des services bilingues à la clientèle. De ce fait, ceux qui cherchent un travail de ce genre, peuvent l'obtenir avec l'aide de cet organisme. Par contre, ceux qui visent un travail plus qualifié ou encore qui veulent continuer dans leur domaine professionnel, ne peuvent pas compter sur le Franco-club pour y parvenir. De plus, ils s'aperçoivent que leurs diplômes ne sont pas reconnus et qu'ils ne peuvent pas travailler immédiatement dans leur domaine au Canada.

Dans l'extrait qui suit, Michel, un client récemment arrivé d'Haïti, parrainé par sa famille qui réside au Canada, s'est rendu au Franco-club avec des besoins spécifiques : «J'étais allé juste pour voir s'ils [le personnel du Franco-club] peuvent m'aider à trouver un emploi [...] Elle [l'intervenante responsable du service de l'emploi] m'a donné des livres et pour que je puisse [...] voir comment je peux évaluer les diplômes que j'ai [...] Parce que j'étais professeur [en Haïti]".

Le discours de cet acteur social explique qu'il utilise uniquement le service d'emploi. Son cas démontre que le concept de "nouvel arrivant " se présente différemment. Il est arrivé comme résident permanent. Il n'a donc pas besoin de services d'immigration. De 
plus, avec l'aide de sa famille, il a eu accès à d'autres ressources, comme à des cours d'anglais et à un médecin.

Amal: Et, est-ce que vous avez trouvé quelque
chose par l'intermédiaire du [Franco-club]?
L'emploi?

Michel : Bon, je ne pourrais pas dire " oui ", parce que jusqu'à présent je ne travaille pas.

Amal : OK, euh, mais vous avez [...] essayé de contacter quelqu'un dans le domaine de l'enseignement ou un organisme?

Michel : Devenir enseignant au Canada [...] c'est très très très difficile [silence] pour moi, c'est très difficile [...] Les difficultés qu'il y a c'est qu'ils demandent tous des choses pour rentrer exactement dans des circuits professoraux quoi.

Cet extrait indique que ce participant n'a pas encore accompli son objectif professionnel. Comme il était enseignant dans son pays pendant une dizaine d'années, il voulait donc continuer à travailler dans son domaine. Il s'est rendu compte que c'est difficile d'y arriver. Sur ce plan, le Franco-club lui a fourni de l'information sur des processus à suivre et des organismes à consulter. Comme il a entrepris lui-même ces démarches auprès des organismes, il trouve que le Franco-club devrait jouer un rôle plus important que cela : "Je pourrais dire qu'ils sont très très courtois [...] et ils vous écoutent. Je pense que c'est peut-être le moyen. Leurs moyens ne sont pas aussi larges pour aider les gens. Mais ils font ce qu'ils pourront faire, quoi ".

Le discours du client indique que l'organisme n'a pas assez de moyens pour répondre à divers besoins de clients. Cet acteur social fait référence à une catégorie de la clientèle comme lui, ceux qui veulent avoir accès à une profession dans un domaine précis. On peut comprendre que, comme il parle de "moyens ", sa vision c'est qu'un organisme comme le Franco-club ne devrait pas se limiter à référer cette catégorie de clients. L'organisme devrait 
"...le Franco-club

offre une gamme

limitée de services

d'emploi en français,

tout en s'appuyant sur

une documentation de

langue anglaise et sur

d'autres organismes

spécialisés opérant en

anglais, tandis que la

population visée attend

de lui la gamme

complète de services en

français. Étant donné que cet organisme est le mieux développé en matière de services d'accueil des nouveaux arrivants opérant en langue française, on peut dire que l'État n'offre pas des services d'appui complets en français. " plutôt intervenir auprès des diverses administrations et associations. Sinon, il devrait aider les clients à obtenir des catégories d'emploi plus diverses. Cependant, son discours soulève le côté courtois qui caractérise la façon dont le service est offert. Il s'agit d'un certain appui humain qu'il a reçu, ce qui ne fait pas partie de la raison d'être du service.

Comme une proportion importante des nouveaux arrivants appartient à cette catégorie, le Franco-club a monté un centre de documentation afin de répondre aux besoins spécifiques de ce groupe. Il s'agit de renseignements sur les normes à suivre comme l'équivalence des diplômes. Le problème qui se pose est que la plus grande partie de la documentation est disponible en anglais. La même intervenante souligne qu' "il y en a en anglais puis il y en a en français, mais il y en a beaucoup en anglais ». De plus, le Franco-club réfere ces clients aux associations et organismes professionnels qui œuvrent dans ce domaine. De même, les organismes qui offrent les services sont souvent anglophones. Dans ce cas, les clients qui ne connaissent pas assez bien l'anglais n'auront pas un accès approprié à ces ressources. Cela indique que ces services ne sont pas entièrement disponibles en français, ni complètement dans des organismes francophones. En somme, le Franco-club offre une gamme limitée de services d'emploi en français, tout en s'appuyant sur une documentation de langue anglaise et sur d'autres organismes spécialisés opérant en anglais, tandis que la population visée attend de lui la gamme complète de services en français. Étant donné que cet organisme est le mieux développé en matière de services d'accueil des nouveaux arrivants opérant en langue française, on peut dire que l'État n'offre pas des services d'appui complets en français.

Ce que cette section démontre premièrement, c'est qu'il y a plusieurs catégories de clients à la recherche d'emplois, ayant divers types le Franco-club offre une gamme limitée de services d'emploi en français, tout en s'appuyant sur une documentation de langue anglaise et sur d'autres organismes spécialisés opérant en anglais, tandis que la population visée attend de lui la gamme complète de services en français. Étant donné que cet organisme est le mieux développé en matière de services d'accueil des 
nouveaux arrivants opérant en langue française, on peut dire que l'État n'offre pas des services d'appui complets en français.

de qualifications et d'objectifs professionnels. Par contre, l'organisme ne peut apporter qu'une aide limitée à certains secteurs spécifiques d'emploi dépendant de l'offre sur le marché de l'emploi. Aussi, tout effort de l'organisme en vue de s'adapter aux besoins réels de sa clientèle est limité par des contraintes étatiques d'ordre administratif et de financement.

Dans les deux types de services que nous venons d'examiner, la question des compétences en langue anglaise est ressortie comme un facteur important, auquel le Franco-club tente de répondre de diverses façons, mais souvent avec pour résultat de se retrouver dans une situation paradoxale étant donné sa raison d'être linguistique, c.-à-d., francophone. Cela mérite un examen plus approfondi. Nous traiterons d'abord des cours d'anglais offerts aux nouveaux arrivants francophones, puis de la place des langues africaines dans l'offre des services.

\section{Les pratiques linguistiques au sein du Franco-club : les cours d'anglais}

Le Franco-club a offert des cours d'anglais langue seconde à l'intention des immigrants pendant plusieurs années dans le cadre d'un programme du gouvernement fédéral. Il s'agissait pour le Franco-club de faire appel aux fonds fédéraux afin de répondre aux besoins des nouveaux arrivants francophones tels qu'identifiés précédemment, tout en créant une opportunité de tisser des liens avec ces francophones dans le but de remplir son rôle de rassembleur.Joscard, l'enseignant qui était responsable des cours d'anglais, explique :

John:Y a-t-il des différences entre ces différents [programmes] si vous voulez? 
"En somme, l'offre de cours d'anglais plaçait le Franco-club devant une foule de contradictions (offrir des cours d'anglais, dont la clientèle n'était pas précisément francophone, tandis que les francophones apprenaient l'anglais ailleurs, tout en mécontentant une catégorie de francophones inéligibles à ces cours d'anglais gratuits) et le Francoclub a cessé ses activités dans ce domaine. »
Joscard : [Un dirigeant du Franco-club] voulait vraiment là [...] qu'ils nous envoient tous les francophones ici. Que ça devienne une école pour francophones [...] On voulait que tous les francophones se sentent un peu comme à la maison [...] C'est toujours plus facile, beaucoup de francophones ensemble, pour le support moral.

De même, ce discours est mondialisant parce qu'il précise que les pratiques linguistiques au sein du Franco-club deviennent de plus en plus bilingues. Cet extrait explique que la direction du Francoclub voulait que les francophones apprennent l'anglais dans un milieu identifié à l'organisme. Ainsi, l'idée du Franco-club est de rassembler les nouveaux arrivants francophones. On remarque aussi qu'il s'agit de créer une sorte de solidarité de groupe et de communauté entre francophones. Dans ce cas, la classe d'anglais devient un regroupement communautaire francophone, un milieu amical et familial. Ce faisant, le Franco-club voulait aussi offrir une plus vaste gamme de services d'établissement aux nouveaux arrivants. Toutefois, du fait qu'il n'existe pas de concentration géographique des francophones à Toronto, les cours étaient offerts dans des locaux dispersés en divers endroits de la ville, au sein d'institutions anglophones. Certains facteurs ont rendu difficile la possibilité de diriger tous les nouveaux arrivants vers ces classes, beaucoup d'entre eux préférant fréquenter des cours d'anglais plus près de leur domicile. Par ailleurs, les cours d'anglais offerts par le Franco-club étaient majoritairement fréquentés par des nouveaux arrivants non francophones. Des frustrations étaient aussi exprimées par des francophones ayant besoin d'apprendre l'anglais, mais qui n'étaient pas éligibles pour les cours d'anglais offerts gratuitement par le Franco-club, ce programme étant réservé aux nouveaux arrivants seulement, compte tenu des règles de financement du gouvernement fédéral.

En somme, l'offre de cours d'anglais plaçait le Franco-club devant une foule de contradictions (offrir des cours d'anglais, dont la clientèle n'était pas précisément francophone, tandis que les francophones apprenaient l'anglais ailleurs, tout en mécontentant 
une catégorie de francophones inéligibles à ces cours d'anglais gratuits) et le Franco-club a cessé ses activités dans ce domaine.Au moment où le Franco-club annulait les cours d'anglais, il appuyait l'introduction des langues africaines dans ses services.

\section{Les langues africaines}

L'un des services, celui d'établissement, est offert, en plus du français, en deux langues africaines, le lingala et le swahili. Cela a été identifié par le gouvernement fédéral comme un besoin afin de fournir un meilleur service aux clients qui parlent ces deux langues comme premières langues. Florence, la conseillère qui dirige ce service est d'origine africaine. Elle mentionne que ces langues sont utilisées : "Pour ceux qui ont de la difficulté à s'exprimer en français [...] Je lui parle directement en lingala et tu vois que c'est un soulagement pour la personne. [...] Quand ils [les clients] savent qu'il y a quelqu'un qui peut s'exprimer en leur langue, ça leur inspire confiance ".

Le discours de cette conseillère est mondialisant du fait qu'il reflète bien les identités et pratiques linguistiques multiples des clients et du personnel du Franco-club. Ce discours explique que les clients qui ne maitrisent pas le français, se sentent plus à l'aise de recevoir le service dans leur langue, car c'est plus facile pour eux. Cependant, ce n'est pas uniquement une question de méconnaissance du français. Même parmi ceux qui s'expriment en français, certains choisissent d'être servis dans leur langue. La même conseillère dit "elle [une cliente] va préférer parler sa langue que de parler en français ». Ainsi, le fait que les clients ont le choix de recevoir le service dans leur langue crée une certaine confiance dans l'organisme. Un lien de rapprochement se crée entre les clients et l'organisme. Il y a institutionnalisation de ces langues au niveau des services. Dans ce sens, les pratiques linguistiques au sein du Franco-club ne sont plus unilingues, ni bilingues (l'anglais est utilisé dans le fonctionnement des services), mais bien multilingues. 
Ayant démontré comment l'organisme s'est adapté aux changements démographiques et aux transformations du rôle de l'État, nous verrons avant de conclure que l'organisme, tout comme l'État, se font une idée du client idéal, mais que cette projection ne correspond pas forcément à la réalité, et que si l'on adopte le point de vue des clients eux-mêmes, il y a une inadéquation entre les services offerts et leurs besoins.

\section{L'organisation des services et les réalités sociales des clients}

Nous donnerons ici l'exemple de Jacques, un Congolais récemment arrivé à Toronto comme demandeur d'asile politique. Quand il est venu au Franco-club, il avait besoin de s'adapter à l'environnement social et d'avoir un aperçu du fonctionnement des institutions. Il voulait aussi comprendre les démarches à suivre au niveau de processus légaux d'immigration.

"J'ai pu bénéficier [...] On m’a fourni certaines adresses qui, en fait, étaient des ressources francophones [...] des écoles de langue par exemple... et certains problèmes de la vie courante [...] Il s'est passé un certain nombre d'ateliers [...] qui ont rencontré mes préoccupations [...] Et il $y$ avait aussi l'aspect [...] institutionnel [...] C'est [...] fabuleux ce qui a été fait [...]J'ai assisté à un atelier où Maître [nom] [...] a parlé de l'immigration [...] dans les aspects les plus divers, par rapport au travail [...] aux études."

Cet extrait démontre que ce client a utilisé une panoplie de services. Sa participation à divers ateliers lui a permis de connaître la société, d'établir des contacts avec les gens et de connaitre le fonctionnement des institutions. Il a eu recours à d'autres services comme l'aide d'interprétariat. Le centre lui a aussi fourni des ressources pour le choix d'un avocat francophone et une école de langue anglaise à côté de chez lui. Ainsi, nous remarquons 
"Finalement, je me suis trouvé un emploi par moimême [...] J'ai surfé le net, j'ai envoyé beaucoup de C.V. Mais le [Franco-club] a, disons, remonté le moral de cette façon pendant que j'étais déprimée. Un genre d'aide psychologique qu'ils m'ont offert pas seulement financier, mais beaucoup psychologique. " que ce client a utilisé plusieurs services du Franco-club. Il s'agit en fait de tous les services dont il a besoin à cette étape de son cheminement comme nouvel arrivant. Il reconnaît l'appui que le Franco-club lui a fourni. Cela s'observe dans son discours par l'emploi des termes comme "j'ai bénéficié ", "c'est intéressant ", et "c'est fabuleux".

"Le [Franco-club] [...] c'était comme une deuxième famille [...] un deuxième volet de l'aspect parental qui m'a aidé à mettre pied sur Toronto [...] Les contacts répétés ont fini par créer d'autres aspects de relation On est plus proches maintenant les uns les autres."

Ce participant fait référence au Franco-club comme à une famille. En plus du lien professionnel, ce discours reflète le rapport amical qu'il a développé envers cet organisme. Cela ne fait pas partie des objectifs du service. En fin de compte, ce client a employé les services en suivant l'ordre établi par l'État et l'organisme. Il n'est pas encore arrivé au service de l'emploi. Il correspond donc à une sorte du client "idéal ", comme prévu par l'État. Par contre, cela ne s'applique pas aux nouveaux arrivants qui optent pour d'autres priorités, surtout l'emploi comme nous le verrons un peu plus loin.

À cet effet, Sylvie-Anne, l'intervenante responsable du service d'emploi souligne l'importance de comprendre la priorité et le contexte social des clients. Cela se relie au soutien moral et émotionnel dont certains clients parlent de façon remarquable. Hélène, une cliente mauricienne, explique son expérience.

"Finalement, je me suis trouvé un emploi par moimême [...] J'ai surfé le net, j'ai envoyé beaucoup de C.V.Mais le [Franco-club] a, disons, remonté le moral de cette façon pendant que j'étais déprimée. Un genre d'aide psychologique qu'ils m'ont offert pas seulement financier, mais beaucoup psychologique."

Cette participante est venue au Franco-club pour utiliser le service d'emploi. Comme elle avait récemment perdu son travail, elle 
traversait une période difficile. Elle dit qu'elle était déprimée. Comme l'explique son discours, elle a finalement trouvé du travail elle-même. Toutefois, elle souligne surtout le soutien moral et psychologique que le Franco-club lui a offert. De ce fait, il s'agit de l'appui moral qui ne fait pas partie de la vision de l'État, ni du mandat de l'organisme qui offre le service. Par contre, la réalité sociale démontre que ce support est important pour les clients ainsi que pour ceux qui offrent les services. Cela peut s'expliquer du fait que la clientèle du Franco-club est en majorité à un stade qui se caractérise par des obstacles comme le dépaysement, la rupture familiale et l'incertitude à l'égard de l'obtention des papiers. Cette étape de la trajectoire migratoire est complexe, elle s'accompagne de sentiments de stress et de dépression. Sur ce plan, le soutien moral devient essentiel.

Comme mentionné précédemment, d'autres clients ne correspondent pas à la norme élaborée par l'État et par l'organisme. Dans ce cas, les services ont plus de mal à s'adapter aux besoins du client. Sylvie-Anne, l'intervenante responsable du service d'emploi soulève un cas qui reflète cet aspect :

Sylvie-Anne : Habituellement ils [les clients] passent par [...] le service d'orientation. Ça m'est arrivé la semaine passée. J'ai eu quelqu'un d'assez nouveau, c'était beaucoup plus délicat.

Amal : Pourquoi?

Sylvie-Anne: J'ai commencé un petit peu, j'ai dit non, ce n'est pas possible [...] Puis là,j'ai changé complètement de discours. Là j'ai dû [dire] ici c'est le service d'aide à l'emploi. C'est comme le deuxième pas, parce que vous n'êtes pas passé par le service d'accueil ou d'adaptation à l'environnement [...] Et je lui ai parlé du service d'accueil [...] Là, il se sentait encore obligé d'expliquer sa situation. C'était difficile pour lui [...] J'ai continué avec lui, tranquillement, à l'introduire aux services d'aide à l'emploi. Parce que pour lui, le besoin c'est tout de suite se trouver un emploi parce 
"Les nouveaux arrivants devraient débuter par le service comme l'accueil, l'orientation et le logement. Dans un deuxième temps, ils seront dirigés au service à l'emploi. Par contre, la réalité sociale de certains clients engendre des besoins qui divergent de ce plan.» qu'il $y$ a des pressions dans sa famille. Alors, il y a [...] tout un monde à analyser [...] toute personne a $[\ldots]$ différents besoins $[. .$.$] priorités.$

Dans ce discours, l'intervenante explique le service tel qu'organisé par le Franco-club en rapport avec les contraintes imposées par l'État qui le finance. Les nouveaux arrivants devraient débuter par le service comme l'accueil, l'orientation et le logement. Dans un deuxième temps, ils seront dirigés au service à l'emploi. Par contre, la réalité sociale de certains clients engendre des besoins qui divergent de ce plan. Le client en question subit des pressions familiales urgentes et veut utiliser le service de l'emploi directement. De ce fait, la priorité pour lui n'est ni l'orientation ni l'apprentissage de l'anglais, mais plutôt l'emploi. Dans ce cas, la contradiction est ici entre la priorité des clients et l'organisation du service.

Ainsi, si certains clients répondent aux critères établis par l'organisme, contraint lui-même par l'État qui le finance, d'autres personnes faisant appel au Franco-club ont d'autres attentes et d'autres besoins qui soit ne sont pas satisfaits, soit reçoivent un appui qui se situe en dehors des obligations imposées et financées par l'État. Ces besoins et ces attentes sont remplis grâce à une certaine forme de bonne volonté des agents communautaires ou institutionnels.

\section{Conclusion}

Dans cet article, nous avons analysé comment le Francoclub, organisme francophone en milieu minoritaire, s'est adapté au changement social et à la mondialisation en se transformant d'un centre culturel ayant pour mission de servir de point de ralliement d'une communauté conçue comme homogène vers un centre d'accueil qui offre des services à une clientèle désormais hétérogène, avec pour conséquence que l'élément de définition linguistique doit être révisé tant en termes de pratiques 
"Ainsi, sur une trentaine d'années, le Franco-club a modifié son mandant d'un organisme qui joue un rôle symbolique de ralliement et de représentation à un guichet de services aux nouveaux arrivants. Le Franco-club doit composer avec une communauté de minorité linguistique se caractérisant par la diversité linguistique ayant de multiples pratiques identitaires et langagières. » langagières individuelles et institutionnelles, qu'en termes de symbole d'identification. Ainsi, sur une trentaine d'années, le Franco-club a modifié son mandant d'un organisme qui joue un rôle symbolique de ralliement et de représentation à un guichet de services aux nouveaux arrivants. Le Franco-club doit composer avec une communauté de minorité linguistique se caractérisant par la diversité linguistique ayant de multiples pratiques identitaires et langagières. Il y a passage du discours modernisant au discours mondialisant.

L'analyse de discours, comme approche théorique et méthodologique, nous a permis d'examiner la construction des réalités sociales de divers points de vue nuancés, que ce soit de celui des citoyens ayant des besoins divers, ou des prestataires de services reliés à l'État ayant un mandat très précis, des utilisateurs de services, ou des employés d'organismes communautaires. Cette analyse révèle des contradictions entre la vision de l'État et des organismes communautaires, d'une part et d'autre part, les réalités sociales des acteurs sociaux, tantôt vus comme membres de communautés linguistiques, tantôt comme de simples usagers de services. C'est la situation paradoxale dans laquelle ces organismes francophones sont désormais placés.

Il a été démontré dans plusieurs études que dans ce contexte de transformation sociale, des processus et des phénomènes d'exclusion sont à l'œuvre. Une étude de Sadiq (2004) a mis en lumière que des organismes des services d'établissement pour les nouveaux arrivants et immigrants de langue anglaise rencontrent des difficultés pour obtenir du financement de l'État, un phénomène également présent chez les organismes d'immigrants de langue française. Par exemple, Madibbo (2004) a documenté le sentiment d'exclusion des immigrants au sein des organismes francophones vis-à-vis leur représentation aux paliers décisionnels. Comme organisme francophone en milieu anglo-dominant, le Francoclub se trouve lui-même en situation d'exclusion linguistique. De même, les clients francophones pourraient aussi subir l'exclusion linguistique ou raciale. Reconnaissant l'existence de tels processus et phénomènes, l'objectif de cet article est néanmoins de mieux comprendre d'autres mécanismes d'exclusion et d'inclusion 
à l'œuvre par lesquels les réalités sociales et démographiques évoluent et des institutions en milieu minoritaire se transforment pour s'adapter à ces nouvelles réalités. Le cas du Franco-club nous montre que l'existence même de l'organisme et le maintien de sa légitimité nécessitent qu'il se redéfinisse dans le sens de l'inclusion de nouvelles clientèles hétérogènes ayant des besoins propres.

\section{Bibliographie}

BER GER, Marie-Josée (1997). "À la recherche d'une intégration : rêves ou réalité ", dans Reflets, Revue ontaroise d'intervention sociale et communautaire, vol. 3, no 2, 75-86.

CHAMBON,Adrienne, Normand Labrie, Monica Heller,Amal Madibbo et John Maury (2001). « L'immigration et la communauté franco-torontoise ", rapport final, centre de recherches en éducation franco-ontarienne, Institut d'études pédagogiques de l'Ontario, Université de Toronto.

FOUCAULT, Michel (1969). L'archéologie du savoir, Bibliothèque des sciences humaines, Paris, Les Éditions Gallimard.

HELLER, Monica et Normand Labrie (2003). «Langue, pouvoir et identité : une étude de cas, une approche théorique, une méthodologie ", dans M. Heller et N. Labrie dir., Discours et identités, la francité canadienne entre modernité et mondialisation, Cortil-Wodon, E.M.E., 9-39.

LABRIE, Normand, Adrienne Chambon, Monica Heller, Fasal Kanouté, Amal Madibbo et John Maury (2002). "Migrations et santé : pratiques des communautés francophones à Toronto ", dans Bulletin suisse de linguistique appliquée, $\mathrm{n}^{\circ} 74,209-235$

MADIBBO, Amal (2004). Minority within a Minority: Black Francophones of Ontario and the Dynamics of Power and Resistance, thèse de doctorat, Institut d'études pédagogiques de l'Ontario. Université de Toronto.

MADIBBO, Amal et John Maury (2002). «L'immigration et la communauté franco-torontoise : le cas des jeunes ", dans Francophonies d'Amérique, n 12,113-122.

MONDADA, Lorenza (1998). "L'identité sexuelle comme accomplissement pratique ", dans : M. J. Fernandez dir., Parler femme en Europe, la femme, image et langage, de la tradition à l'oral quotidien, Paris et Montréal, L'Harmattan, 253-276.

SADIQ, Kareem ( 2004). "The Two-Tier Settlement System : A Review of current New Comer Settlement Services in Canada”, CERIS Working Paper Series, no. 34, Toronto, Joint Centre of Excellence for Research on Immigration and Settlement.

\section{Notes}

1. Tous les noms ont été remplacés par des pseudonymes.

2. Le projet L'immigration et la communauté franco-torontoise a été subventionné par le Joint Centre of Excellence for Research on Immigration and Settlement / Toronto. L'étude a été menée entre 1999-2001au Centre de recherches en éducation franco-ontarienne de l'université 
de Toronto. L'équipe de recherche était formée des chercheurs principaux Monica Heller et Normand Labrie (OISE/UT),Adrienne Chambon (Faculté de travail social/UT), Fasal Kanouté (Université de Montréal) et des assistants de recherche Amal Madibbo, Mueni Malubungi (OISE/UT), et John Maury (Département d'études françaises/UT). 